\title{
dspace.vutbr.cz
}

\section{Fractional-Order Hartley Oscillator}

AGAMBAYEV, A.; KARTCI, A.; HASSAN, A. H.; HERENCSÁR, N.; BAGCI, H.; SALAMA, K. N.

Proceedings of the 2018 14th Conference on PhD Research in Microelectronics and Electronics (PRIME) pp. 45-48

elSBN: 978-1-5386-5387-6

DOl: http://dx.doi.org/10.1109/PRIME.2018.8430336

Accepted manuscript

(C2018 IEEE. Personal use of this material is permitted. Permission from IEEE must be obtained for all other uses, in any current or future media, including reprinting/republishing this material for advertising or promotional purposes, creating new collective works, for resale or redistribution to servers or lists, or reuse of any copyrighted component of this work in other works. AGAMBAYEV, A.; KARTCI, A.; HASSAN, A. H.; HERENCSÁR, N.; BAGCl, H.; SALAMA, K. N., " Fractional-Order Hartley Oscillator", Proceedings of the 2018 14th Conference on PhD Research in Microelectronics and Electronics (PRIME), pp. 45-48, 2018. DOI: 10.1109/PRIME.2018.8430316. Final version is available at https://ieeexplore.ieee.org/document/8430336/ 


\title{
Fractional-Order Hartley Oscillator
}

\author{
Agamyrat Agambayev ${ }^{1}$, Aslihan Kartci ${ }^{2}$, Ali H. Hassan ${ }^{1}$, Norbert Herencsar ${ }^{2}$, Hakan Bagci ${ }^{1}$, and Khaled N. Salama ${ }^{1}$ \\ ${ }^{1}$ Computer, Electrical and Mathematical Science and Engineering (CEMSE) Division, King Abdullah University of Science and Technology \\ (KAUST), Thuwal 23955, Saudi Arabia \\ ${ }^{2}$ Faculty of Electrical Engineering and Communication, Brno University of Technology, Brno, Czech Republic \\ agamyrat.agambayev@kaust.edu.sa, kartci@feec.vutbr.cz, ali.h.hassan@ieee.org, herencsn@feec.vutbr.cz, hakan.bagci@kaust.edu.sa,
} khaled.salama@kaust.edu.sa

\begin{abstract}
A fractional-order capacitor (FOC) is developed using a Molybdenum disulfide ferroelectric polymer composite. The fabricated FOC exhibits constant phase over five decades between $100 \mathrm{~Hz}-10 \mathrm{MHz}$, which is the broadest operating frequency bandwidth reported so far for an FOC. Furthermore, a fractional-order Hartley oscillator is built using this FOC, and provide ten times higher oscillation frequency than the frequency of the conventional Hartley oscillator counterpart.
\end{abstract}

Keywords-constant phase element, FOC, fractional-order capacitor, Hartley oscillator

\section{INTRODUCTION}

Although, the three basic passive circuit elements, namely, capacitor, resistor, and inductor have been around for three centuries, understanding and design of classical resistorcapacitor, and resistor-inductor, and capacitor-inductor, and resistor-capacitor-inductor circuits have remained fundamentally unaltered, since only integer-order differential equations are used in circuit design [1]. However, great improvements in the study of fractional calculus that deals with fractional-order derivation and integration have been achieved over the last five decades [2]. In the meantime, the fractionalorder generalization has been used for circuit/device design and modeling [2] in various field such as biomedicine [3-4], energy-storage and generation [5], agriculture [6], electromagnetics [7], and control systems [8].

Recently, different approaches to realization of fractionalorder elements (called constant phase elements in electrochemistry), particularly, fractional-order capacitors (FOCs) [9-13] have been developed. Among these approaches, those make use of composites of different materials have gained traction, since they lead to FOCs that can be easily integrated with microelectronics, and design an FOC with tunable constant phase angle (CPA) [9-10]. However, their narrow band of operating frequency (i.e., maximum two decades) limited their use in building circuits such as filters and oscillators with fractional-order transfer functions. It is also extremely important to develop an FOC with a constant phase zone (CPZ), where FOC exhibits a CPA with only small variation, in the order of $\pm 4^{\circ}$ over the full band of operation.

The impedance of an FOC is given by $Z=1 /\left[(j \omega)^{\alpha} C_{\alpha}\right]$ where $C_{\alpha}$ is the pseudocapacitance (also known as fractance) and $\alpha(0<\alpha<1)$ is the fractional order. The phase angle associated with $Z$ is given by $\phi=-\alpha \pi / 2$. Table I compares
TABLE I. IMPEDANCE, ORDER, AND PHASE ANGLE OF INDUCTOR, RESISTOR, CAPACITOR, AND FOC.

\begin{tabular}{|c|c|c|c|c|}
\hline Components & Inductor & Resistor & FOC & Capacitor \\
\hline Impedance & $Z=j \omega L$ & $Z=R$ & $Z=1 /\left[(j \omega)^{\alpha} C_{\alpha}\right]$ & $1 /(j \omega C)$ \\
\hline$\alpha$ & $\alpha=-1$ & $\alpha=0$ & $0<\alpha<1$ & $\alpha=1$ \\
\hline Phase $(\phi)$ & $\phi=90^{\circ}$ & $\phi=0$ & $0>\phi>-90^{\circ}$ & $\phi=-90^{\circ}$ \\
\hline
\end{tabular}

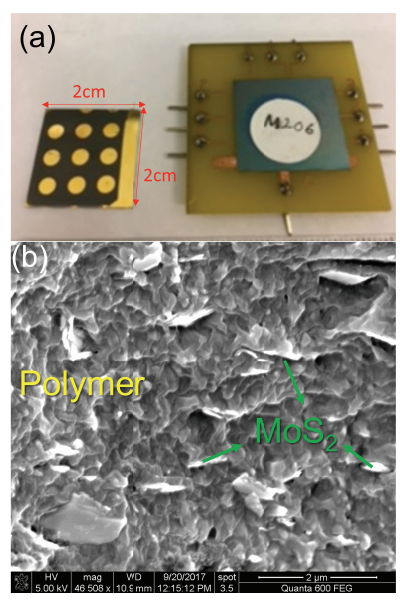

Fig. 1. (a) PCB-compatible FOC fabricated using $\mathrm{MoS}_{2}$-polymer nanocomposite. (b) The cross-sectional SEM image of the nanocomposite.

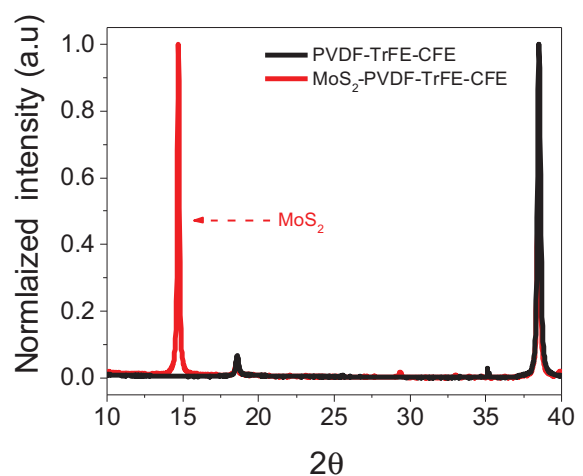

Fig. 2. Normalized XRD patterns of prepared polymer and $\mathrm{MoS}_{2}$-polymer nanocomposite films. 
the frequency dependent impedance, order, and as well as phase angle of passive circuit elements, namely, inductor, resistor, capacitor, and ideal FOC.

In this work, we report on an FOC fabricated using a new class of Molybdenum disulfide $\left(\mathrm{MoS}_{2}\right)$ filled Polyevinelidenefluoride-trifluoroethylene-chlorofluoroethylene (PVDF-TrFE-CFE) composite and exhibits a constant phase over five decades between $100 \mathrm{~Hz}-10 \mathrm{MHz}$. This FOC is then used in building an oscillator, which can, for example, be used for modulating/demodulating frequency between the highspeed radio-frequency (RF) input and the baseband system blocks. The circuit design used here adopts the Hartley oscillator since it has a comparable performance like the other topologies such as CMOS LC and Colpitts oscillators [14-15]. To show the advantages of using an FOC in the design of an oscillator, two Hartley oscillators are built; one with an FOC and other with classical capacitor counterpart, and their oscillation frequencies are compared. Measurement results demonstrate that the output frequency of the fractional-order Hartley oscillator is almost ten times greater than the frequency of its conventional counterpart.

\section{FOC FABRICATION AND CHARACTERIZATION}

First, terpolymer namely, PVDF-TrFE-CFE powder is dissolved in a N, N-Dimethylformamide (DMF) solvent, under magnetic stirring for 48 hours to get $0.1 \mathrm{~g} / \mathrm{ml}$ solution [16]. The $\mathrm{MoS}_{2}$ powder is dispersed in DMF at a concentration of 20 $\mathrm{mg} / \mathrm{ml}$ and stirred for two hours using a tip-type sonicator. 90 $\%$ of the $\mathrm{MoS}_{2}$-DMF solution is collected and DMF is evaporated in a freezer dryer. The resulting $\mathrm{MoS}_{2}$ nanosheets are dispersed again in DMF at the $0.03 \mathrm{~g} / \mathrm{ml}$ concentration using ultra-sonication bath for two hours and mixed with the PVDF-TrFE-CFE solution in proportion by stirring for 24 hours at room temperature. Finally, the mixture is further stirred for one hour using a tip-type sonicator before drop casting. The composite solutions are casted onto a $200 \mathrm{~nm} \mathrm{Au}$ covered $\mathrm{SiO}_{2} / \mathrm{Si}$ substrate and DMF is evaporated for overnight at $110{ }^{\circ} \mathrm{C}$ under vacuum. In order to make the top electrode, $\mathrm{Au}$ circular electrode with $1.5 \mathrm{~mm}$ radius and 200 $\mathrm{nm}$ thickness is deposited onto nanocomposite film using a shadow mask. Nine FOCs are fabricated on a chip with $2 \mathrm{~cm} \times$ $2 \mathrm{~cm}$ area. The chip is bonded using the silver paste on a printed circuit board $(\mathrm{PCB})$ to characterize and use in electrical circuits.

Fig. 1(a) shows the photograph of the nanocomposite film and the PCB-compatible FOC. The cross-section scanning electron microscopy (SEM) image of the nanocomposite film with $\mathrm{MoS}_{2}$ is shown in Fig. 1(b). The composite presents a compact structure where the $\mathrm{MoS}_{2}$ nanosheets are distributed homogeneously. During the mixing process, the polymer chains wrap the $\mathrm{MoS}_{2}$ nanosheets and avoid the aggregation of $\mathrm{MoS}_{2}$.

Furthermore, Fig. 2 compares the X-ray diffraction (XRD) patterns of the terpolymer and nanocomposite films, namely. PVDF-TrFE-CFE and the $\mathrm{MoS}_{2}$ :PVDF-TrFE-CFE respectively. It is important to note here that the XRD spectra are normalized with respect to peak at $38^{\circ}$ (due to the gold electrode). An intense peak take places at $18.2^{\circ}$ XRD spectrum of the both film which correspond to a (111) plane of PVDFTrFE-CFE. The XRD spectrum of the nanocomposite shows only one additional peak at $15.1^{\circ}$ which belong to the $\mathrm{MoS}_{2}$ nanosheets as expected, and. confirms that extra complicated molecular structures are not formed at the interface of polymer$\mathrm{MoS}_{2}$ nanosheets.

An Agilent 4994A precision impedance analyzer with the $16048 \mathrm{G}$ fixture is used in electrical characterization of fabricated FOCs. The magnitude and phase angle of the FOCs' impedance are measured. Standard calibration tests (open and short circuits) are performed to calibrate the instrument. Note that the thickness of films used for electrical characterizations is $30 \mu \mathrm{m}$. A total of 801 measurements are taken in the frequency band between $100 \mathrm{~Hz}$ to $10 \mathrm{MHz}$. Fig. 3(a) plots the measured phase angle versus frequency. The figure clearly shows that the phase angle is nearly constant and stable over a broad frequency range. If the maximum error allowed in phase angle is set to $\pm 4^{\circ}$, the CPA is computed (by averaging) to be $-58^{\circ}$ between $100 \mathrm{~Hz}$ and $10 \mathrm{MHz}$ (five decades). This CPA value corresponds to $\alpha=0.64$. The phase angle deviation (the difference between the measured phase angle and the CPA) is plotted versus frequency in the inset in Fig. 3(a).
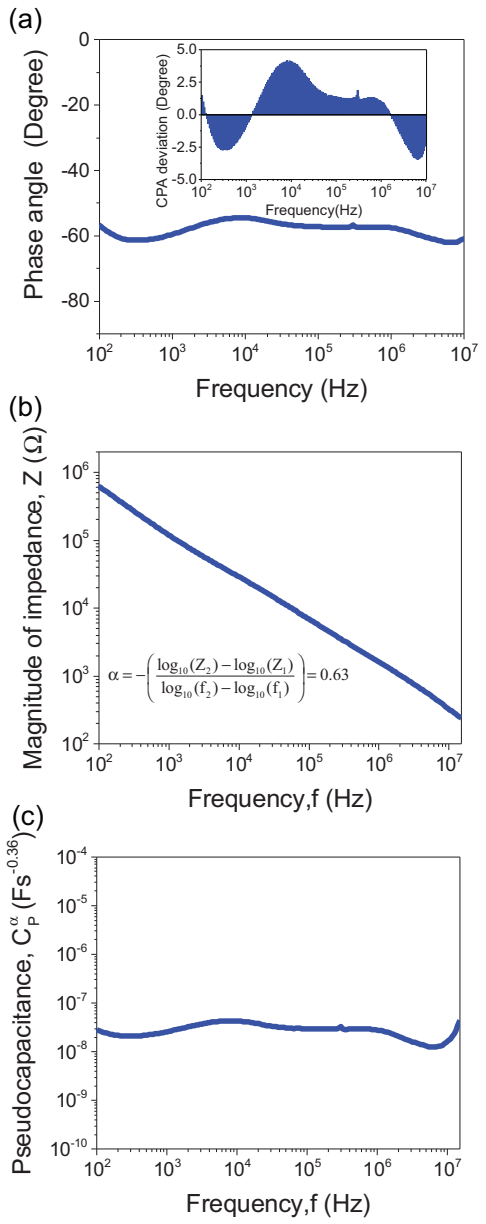

Fig. 3. (a) Measured phase angle versus frequency. (b) Measured magnitude of impedance versus frequency. (c) Pseudocapacitance computed using measured magnitude and phase angle versus frequency. 


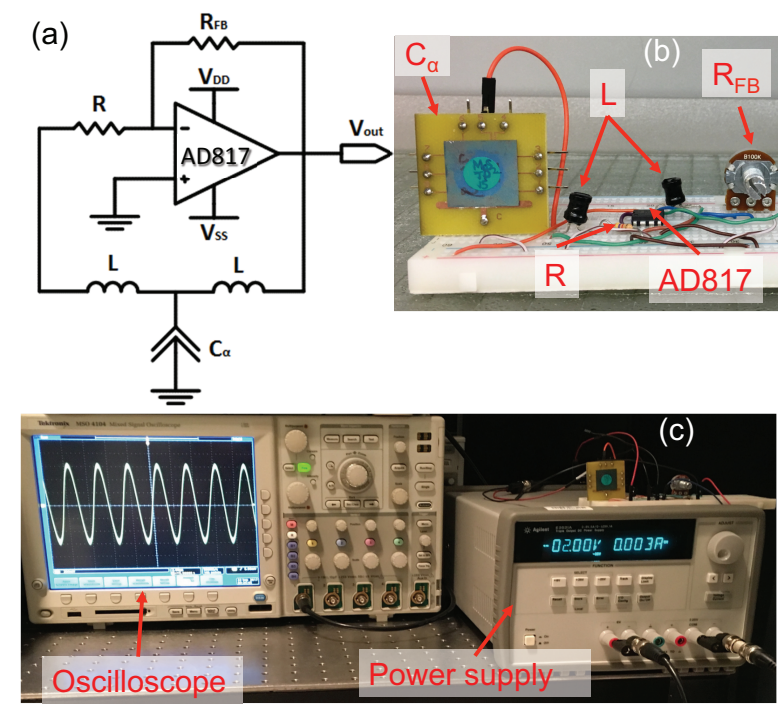

Fig. 4. (a) Hartley oscillator circuit schematic with a fractional-order capacitor (b) Circuit implementation of the Hartley oscillator. (c) Test setup for measuring the output voltage of the oscillator.

Fig. 3(b) plots the measured magnitude of impedance versus frequency. Note that $\log |Z|=-\alpha \log (\omega)-\log \left(\mathrm{C}_{\alpha}\right)$ and $\alpha$ (which is the slope in the above equation) is computed by applying (linear) least-squares fit to the samples of $\log |Z|$ in the defined CPZ. This operation yields $\alpha=0.63$, which confirms the value obtained by averaging the CPA [Fig. 3(a)]. Along $\alpha$, pseudocapacitance $C_{\alpha}$ also plays key role in designing an FOC.

Fig. 3(c) plots $C_{\alpha}=1 /\left(\omega^{\alpha}|Z|\right)$ versus frequency and shows that $C_{\alpha}$ is almost stable over the defined $\mathrm{CPZ}$ with an average value of $28.3 \mathrm{nFs}^{-0.36}$.

\section{FRACTIONAL-ORDER HARTLEY OSCILLATOR}

This section introduces the fractional-order Hartley oscillator [Fig. 4(a)]. The oscillation frequency of this circuit $f_{\text {osc }}$ satisfies the given equation below [17]:

$$
\left(2 \pi f_{\text {osc }}\right)^{1+\alpha}-\frac{(2 r+R) / L}{\tan (\alpha \pi / 2)}\left(2 \pi f_{o s c}\right)^{\alpha}-\frac{2 / L C_{\alpha}}{\sin (\alpha \pi / 2)}=0
$$

where $L$ is the inductance, and $r$ is the internal resistance of the inductors, and $R$ is the resistance of the resistor.

Eq. (1) is solved for $f_{\text {osc }}$ for values of $\alpha$ changing between 0.1 and 1 while $C_{\alpha}=28.3 \mathrm{nFs}^{-0.36}, R=1 \mathrm{k} \Omega, L=1 \mathrm{mH}$, and $r=15 \Omega$. Fig. 5 plots $f_{\text {osc }}$ versus $\alpha$. The figure shows that $f_{\text {osc }}$ increases as $\alpha$ is decreased. As expected, the relation is nonlinear. Especially for small values of $\alpha$, a small change in $\alpha$ results in a large change in $f_{\text {osc }}$. Note that setting $\alpha=1$ in Eq. (1) provides the oscillation frequency of the conventional Hartley oscillator.

The fractional-order Hartley oscillator is built using the fabricated FOC, the resistor with $R=1 \mathrm{k} \Omega$, the inductors with
$L=1 \mathrm{mH}$ and $r=15 \Omega$, and a commercial AD 817 operational amplifier with $\pm 2 \mathrm{~V}$ supplies [Figs. 4(a) and (b)]. In addition, the feedback resistor $R_{F B}$ is equal to $100 \mathrm{k} \Omega$ potentiometer. Fig. 4(c) shows the test set up for Hartley oscillator.

By solving Eq. (1) with these parameters, the oscillation frequency is calculated to be $805.8 \mathrm{kHz}$ for the fractional-order oscillator. For the oscillator with the conventional capacitor ( $\alpha=1$ ), the oscillation frequency is found to be $82.8 \mathrm{kHz}$. These calculated values are in close agreement with the measured output frequencies $793.9 \mathrm{kHz}$ and $81.1 \mathrm{kHz}$ respectively (Fig. 6). The output voltage swing is constant around $2.18 \mathrm{~V}_{\mathrm{pp}}$ for both oscillators. Finally, the measured results show that oscillator with the fabricated FOC with $\alpha=0.64$ exhibits a higher oscillation frequency. It is almost ten times of the frequency of the oscillator with the conventional capacitor $(\alpha=1)$.

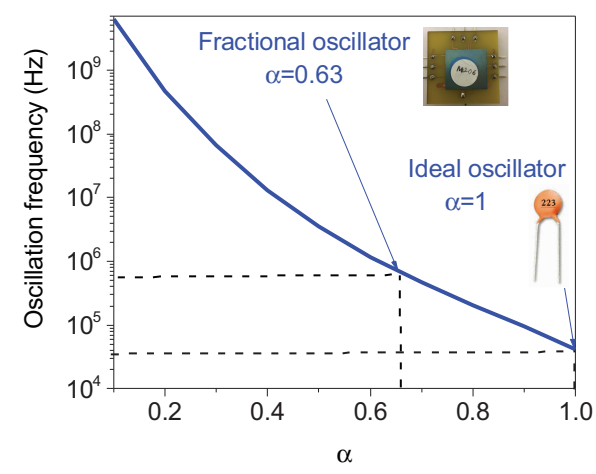

Fig. 5. Calculated oscillation frequency versus $\alpha$. The chip connected to PCB contains nine individual FOCs.

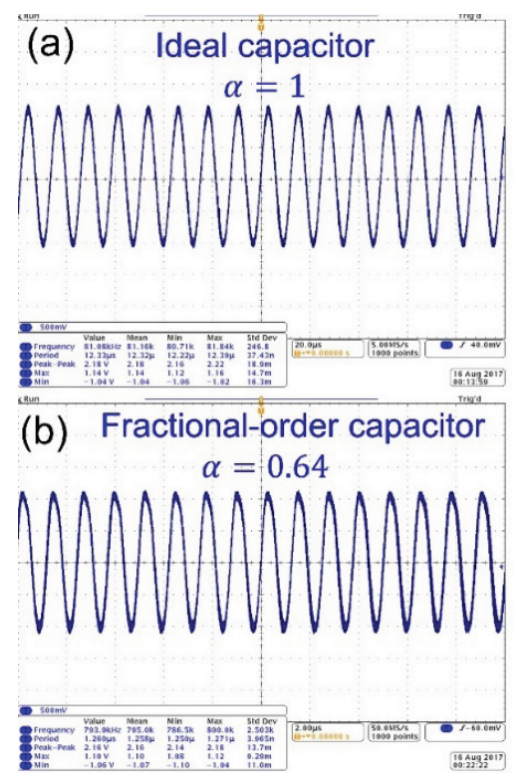

Fig. 6. The measured output voltage of the Hartley oscillator for (a) $\alpha=1$ (b) $\alpha=0.64$. 


\section{CONCLUSION}

$\mathrm{MoS}_{2}$ reinforced PVDF-TrFE-CFE is used to fabricate an FOC. A straightforward solution-mixing approach to generate the nanocomposites films makes easy the tailoring of the electrical properties of the resulting FOCs. The cross-sectional SEM image of the composite confirms that $\mathrm{MoS}_{2}$ is distributed uniformly inside PVDF-TrFE-CFE without forming an aggregation. Moreover, the structural composition of the PVDF-TrFE-CFE film and the $\mathrm{MoS}_{2}$ :PVDF-TrFE-CFE composite are investigated using XRD. Only specific peaks associated with each constituent are observed in XRD spectrum showing that unexpected molecular structure is not formed at the $\mathrm{MoS}_{2}$-polymer interface.

The FOC fabricated using the $\mathrm{MoS}_{2}$ :PVDF-TrFE-CFE nanocomposite has a $\mathrm{CPA}$ of $-58^{\circ}$ within the $\mathrm{CPZ}$ changing from $100 \mathrm{~Hz}$ to $10 \mathrm{MHz}$ (five decades). This is significantly broader than the $\mathrm{CPZ}$ of the previously reported nanocomposite FOCs, which changes from $50 \mathrm{kHz}$ to $10 \mathrm{MHz}$.

A fractional-order Hartley oscillator is designed employing the fabricated FOC. Measurements and analytical predictions show that oscillation frequency of the fractional-order Hartley oscillator is almost ten times than the oscillation frequency of the conventional Hartley oscillator counterpart.

In summary, we believe that FOCs from $\mathrm{MoS}_{2}$-PVDF based polymers nanocomposites paves the way for novel fractional-order electronic systems [18-21].

\section{REFERENCES}

[1] A. Kartci, A. Agambayev, N. Herencsar, and K. N. Salama, "Series-, parallel-, and inter-connection of solid-state arbitrary fractional-order capacitors: theoretical study and experimental verification," IEEE Access, vol. 6, pp. 10933-10943, 2018.

[2] A. S. Elwakil, "Fractional-order circuits and systems: An emerging interdisciplinary research area," IEEE Circuits and Systems Magazine, vol.10, pp.40-50, 2010.

[3] T. C. Doehring, A.H. Freed, E.O. Carew, and I. Vesely, "Fractionalorder viscoelasticity of the aortic valve: an alternative to QLV," J. Biomech. Eng., vol. 127, pp. 700-708, 2005.

[4] T. J. Freeborn, "A survey of fractional-order circuit models for biology and biomedicine," IEEE Journal on emerging and selected topics in circuits and systems, vol. 3, pp: 416-424, 2013.

[5] M. E. Fouda, A. S. Elwakil, A. G. Radwan, and A. Allagui, "Power and energy analysis of fractional-order electrical energy storage devices," Energy, vol. 111, pp: 785-792, 2016.
[6] S. Jesus, T. J. A. Machado, and B. J. Cunha, "Fractional electrical impedances in botanical elements," J. Vib. Control 14, pp. 1389-1402, 2008

[7] F. L. Oustaloup, and B. Mathieu, "Frequency-band complex noninteger differentiator: characterization and synthesis," IEEE Trans. Circuits Syst. I, Fundam. Theory Appl., vol. 47, pp. 25-39, 2000.

[8] G.W. Bohannan, "Analog fractional-order controller in temperature and motor control applications," Journal of Vibration and Control, vol. 4, pp. 1487-1498, 2008

[9] A. M. Elshurafa, M. N. Almadhoun, K. N. Salama, and H. N. Alshareef, "Microscale electrostatic fractional capacitors using reduced graphene oxide percolated polymer composites," Applied Physics Letters, vol. 102, no. 23, pp. 232901, 2013.

[10] D. A John, S. Banerjee, G. W Bohannan, and K. Biswas, "Solid-state fractional capacitor using MWCNT-epoxy nanocomposite," Applied Physics Letters, vol. 110, no. 16,pp.163504, 2017.

[11] A. Agambayev, K. H. Rajab, A. H. Hassan, M. Farhat, H. Bagci, and K. N. Salama, "Towards fractional-order capacitors with broad tunable constant phase angles: Multi-walled carbon nanotube-polymer composite as a case study," J. of Physics D: App. Physics, vol. 51, pp. 1-6, 2018.

[12] A. K. Gil'mutdinov, P. A. Ushakov, and R. El-Khazali, Fractal Elements and their Applications. Springer, 2017.

[13] A. Agambayev, S. Patole, H. Bagci, and K. N. Salama, "Tunable fractional-order capacitor using layered ferroelectric polymers," AIP Advances, vol. 7, no. 9, pp. 095202, 2017.

[14] I. Chlis, D. Pepe, and D. Zito, "Comparative analyses of phase noise in $28 \mathrm{~nm}$ CMOS LC oscillator circuit topologies: Hartley, Colpitts, and common-source cross-coupled differential pair," The Scientific World Journal, vol. 2014, 2014.

[15] A. Kartci, N. Herencsar, J. Katon, L. Brancik, K. Vrba, G. Tsirimokou and C. Psychalinos. "Fractional-order oscillator design using unity-gain voltage buffers and OTAs," IEEE International Midwest Symposium on Circuits and Systems (MWSCAS), Boston, USA, pp. 555-558, 2017.

[16] A. Agambayev, S. Patole, M. Farhat, A. Elwakil, H. Bagci, and K. N. Salama, "Ferroelectric fractional-order capacitors," ChemElectroChem, vol. 4, pp. 2807-2813, 2017.

[17] A. S Elwakil, A. Agambayev, A. Allagui, K. N. Salama, "Experimental demonstration of fractional-order oscillators of orders 2.6 and 2.7," Chaos, Solitons \& Fractals, vol. 96, pp. 160-164, 2017.

[18] S. Elmetennani, I. N'Doye, K. N. Salama, and T. M. Laleg- Kirati, "Performance analysis of fractional-order PID controller for a parabolic distributed solar collector," IEEE AFRICON, pp. 440-445, 2017.

[19] I. N'Doye, K. N. Salama, and T. M. Laleg-Kirati "Robust fractionalorder proportional-integral observer for synchronization of chaotic fractional-order systems," IEEE/CAA Journal of Automatica Sinica, pp.1-10, 2018.

[20] A. Tepljakov, E. Petlenkov, J. Belikov, and M. Halas, "Design and implementation of fractional-order PID controllers for a fluid tank system," American Control Conference (ACC), pp. 1777-1782, 2013

[21] A. G. Radwan and K. N Salama, "Fractional-order RC and RL circuits," Circuits, Systems, and Signal Processing, vol. 31, pp. 1901-1915, 2012. 